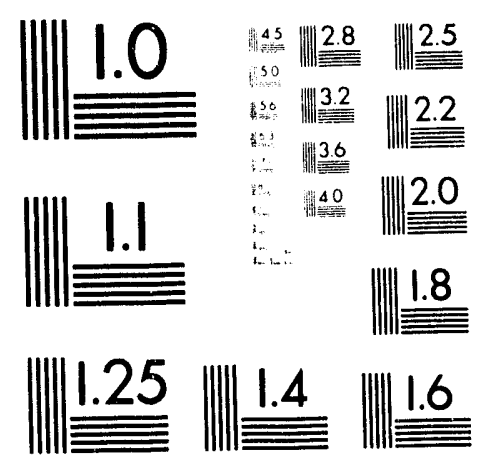



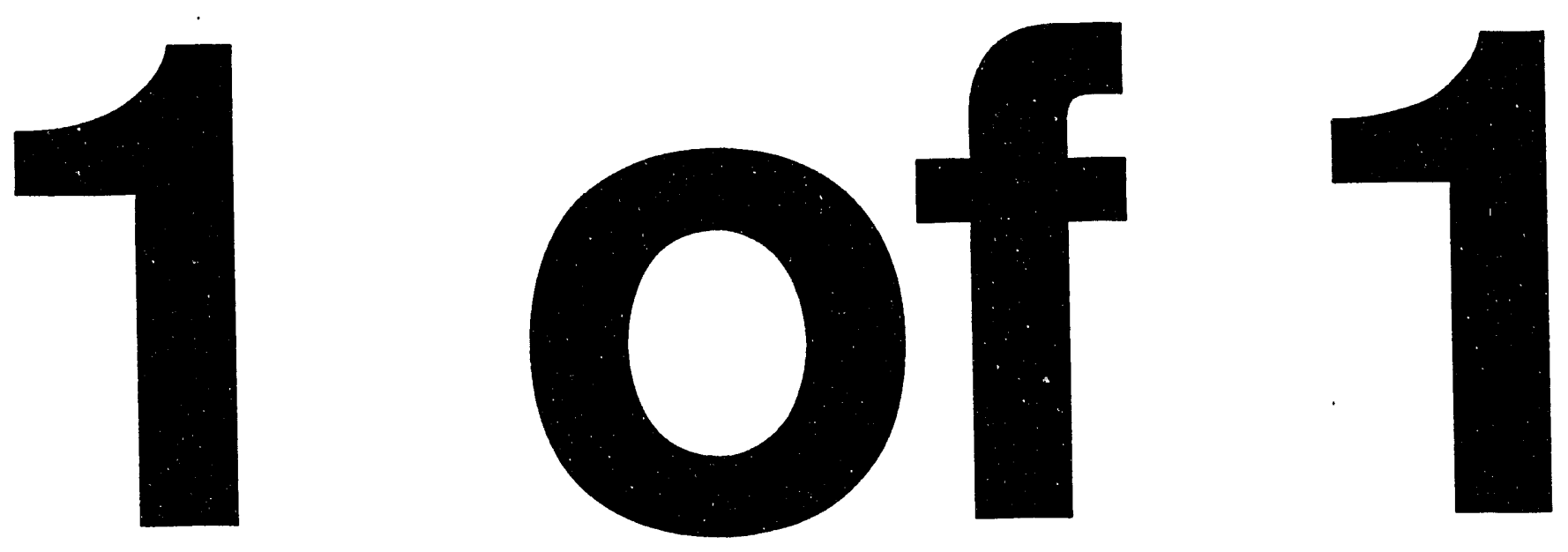


\title{
An Injection Scheme for the Brookhaven ATF Utilizing Space-Charge Emittance Growth Compensation
}

Juan C. Gallardo and Harold G. Kirk Physics Department, 901.A

May 1993

\section{CENTER FOR ACCELERATOR PHYSICS}

\author{
BROOKHAVEN NATIONAL LABORATORY \\ ASSOCIATED UNIVERSITIES, INC. \\ Under Contract No. DE-AC02-76CH00016 with the \\ UNITED STATES DEPARTMENT OF ENERGY
}




\section{DISCIAIMER}

This report was prepared as an account of work sponsored by an agency of the (Inited States Government. Neither the United States Government nor any agency thereof, nor any of their employees, nor any of their contractors, subcontractors, or their employees, makes any warranty, express or implied, or assumes any legal liability or responsibility for the accuracy, completeness, or usefulness of any information, apparatus, product, or process disclosed, or represents that its use would not infringe privately owned rights. Reference herein to any specific commercial product, process, or service by trade name, trademark. manufacturer, or otherwise, does not necessarily constitute or imply its endorsement, recommendation, or fa voring by the United States Government or any akency, contractor or subcontractor thereof. The views and opinions of authors expressed herein do not necessarily state or reflect these of the United States Government or any agency, contractor or subcontractor thereof. 


\title{
AN INJECTION SCHEME FOR THE BROOKHAVEN ATF U'TILIZING SPACE-CHARGE EMITTANCE GROWTH COMPENSATION
}

\author{
Juan C. Gallardo and Harold G. Kirk \\ Physics Department \\ Brookhaven National Laboratory \\ Upton, New York 11973
}

\begin{abstract}
We consider possible configurations for injection of photocathode-produced electron beams straight into linear accelerating sections. A slightly convergent beam into the linac is required for maximum transmission through the linac sections and therefore a solenoid pair is considered. We describe strategies for placing the rf gun injector and the strength of the solenoids so as to minimixe the emittance growth of the bearn as it traverses the linac sections. The simulation codes PARMELA and TRACE 3-D were used for this design study.
\end{abstract}

\section{INTRODUCTION}

The Brookhaven Accelerator Test Facility consists of a photocathode loaded rf gun, a double-bend, low-energy transport system, two traveling-wave accelerating sections and a high-energy transport to an experimental hall [1]. Our goal in this paper is to examine beam line configurations which will permit straight injection into the linac sections without having to resort to bending magnets. The control of emittance growth is a primary design objective.

The issue of emittance growth and containment in high-brightness electron beams has been studied extensively. It has been argued that to study the physical phenomena that produces emittance dilution in the transport and acceleration of a high-brightness electron beam it is desirable to divide the electron bunch in longitudinal slices. These slices provide a tool to probe and extract the different dynamical behavior of the slices of the electron pulse. It is well known that emittance dilution due to the rf dynamics within the of gun [2] is quite different from that due to space charge [3] within the bunch structure of the electron beam.

Using the code PARMELA [4], we track each slice through the system and observe that the intrinsic emittance within each slice can be very small $\left(\epsilon_{n} \approx 1 \pi \mathrm{mm}\right.$ mrad) but the projection on the phase space of all slices give rise to a larger global emittance. Although, the area in phase space (emittance) swept by each slice is small, the area covering all the slices is significantly larger and consequently, the emittance of the total beam is larger in magnitude. Since the space-charge effects are greater at the longitudinal center of the beam than at the ends, the core tends to develop larger values of $\langle\boldsymbol{x}\rangle$ and $\left\langle\boldsymbol{x}^{\prime}\right\rangle$. This leads to each slice evolving differently in phase space as time evolves.

\section{NUMERICAL ANALYSIS AND RESULTS}

\subsection{Long Drift Solution}

Injecting beam straight into the linacs entails placing an if gun inline with the linacs and utilizing a solenoidgun-solenoid arrangement while possibly incorporating the present inline triplet for matching beam into the linac. The solenoid preceeding the gun is used so as to buck the magnetic field of the second solenoid, thus insuring that an emittance degrading magnetic field is not present at the photocathode. In this configuration the cathode plane is located 2.5 meters from the linac entrance. The advantage of this approach is that it preserves the present double-bend for injection into the linac [5] thus providing for valuable redundancy thereby reducing program downtime.

Matching the beam into the linac so as to encounter no beam loss after traversing the two linac sections requires the electron beam to be slightly converging as it enters the first linac stction. We have checked that this is not possible for a $1 \mathrm{nC}, 10 \mathrm{ps}$ beam if we wish to rely exclusively on the focusing of a solenoid. By utilizing the inline triplet, it is possible to present a properly matched beam to the linac. We find, however, that a solenoid placed immediately after the gun is still required in order to contain the beam during the $70 \mathrm{~cm}$ drift to the triplet entrance. We used TRACE 3-D [6] to develop the intial conditions for matching the electron beam into the linac and then used PARMELA to calculate the full beam dynamics from the plane of the photocathode through to the emergence from the two linac sections.

We show in Fig. 1 a typical solution for delivering full beam through the linac. The electron beam parameters for this solution entail beginning at the photocathode with a beam with a uniform radial distribution of $r=$ $0.9 \mathrm{~mm}$ and a uniform longitudinal pulse length of 10 ps. The use of a uniform distribution was found to offer significant advantages over gaussian distributions in terms of minimizing the final beam emittance. The beam emerges from the linac sections with an rms radius of 1.6 $\mathrm{mm}$ and a transverse emittance of $3.5 \pi \mathrm{mm}$-mrad.

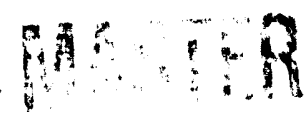

DISTRIBUTION OF THHS DOCUMENT IS UNLIMITED

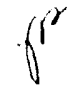




\section{2 Close-in Solution}

We explore placing the rf gun inline as close as possible to the linac entrance. Matching into the linac, however, requires at a minimum a solenoidal focusing magnet between the gun and the linac entrance. The principal variables explored for this study are: the strength of the solenoid field, the gun-to-linac distance, and the phase of the linac sections relative to the of gun. The length of the solenoid was fixed at $16 \mathrm{~cm}$ and the average accelerating gradient of the linac sections was set at $7 \mathrm{MV} / \mathrm{m}$. While we did vary the radius of the initial electron beam, the bunch charge was fixed at $\mathrm{lnC}$ and the bunch length was fixed at 10 ps.

Fig 2 shows the solution of the $z=62 \mathrm{~cm}$ case, presenting a beam identical to the previous example displayed in Fig. 1. The beam emerges from the two linac sections with an rms radius of $028 \mathrm{~mm}$, an rms divergence of $0.050 \mathrm{mrad}$, and an invariant emittance $\epsilon_{n}=0.75 \pi$ $\mathrm{mm}$-mrad. We find that the energy spread of the emerging beam is particularly sensitive to the phasing of the linac sections while the final beam emittance is rather insensitive to this variable (see Fig. 3). The optimum setting of the phase delivers a beam with an $\mathrm{rms} d p / p$ of $2 \times 10^{-3}$. In Fig. 4, we display the results of varving the drift distance between the gun-solenoid and the linac entrance. We find that the optimum distance from the photocathode plane to the linac is $70 \mathrm{~cm}$.

\subsection{Electron Bunch Dynamics}

We have examined the process whereby the emittance of the electron first grows then diminishes as the beam drifts then accelerates after it leaves the if gun. The emittance growth experienced during the drift period immediately after the beam leaves the if gun results from defocusing forces due to space charge within the electron bunch. These defocusing forces are stronger in the center of the bunch than on the leading and trailing edges of the beam. This results in a time-dependent, phase-space structure which can be neutralized if the space charge forces are inverted so as to make the defocusing forces stronger in the ends of the electron bunch than the center. The can be achieved though a focusing action on the beam followed by a drift. In Fig. 5 , we see the trajectories in $x-x^{\prime}$ phase space of an ensemble of electrons first at the longitudinal ends of the bunch and then at the longitudinal center of the bunch. Notice that after the focusing action is complete both ensembles move toward the left (toward negative $x$ ) due to drift and simultaneously up (toward positive $x^{\prime}$ ) due to defocusing space-charge forces. The interesting feature observed in this plot is that a cross-over occurs going from negative to positive $x^{\prime}$ (defocusing action) instead of the standard positive to negative $x$ (drift action). Clearly seen in the figure is that the end sections of the electron bunch are spatially compressed more than the center of the bunch. This results in an increase of the space-charge defocusing forces in the ends relative to the center thus compensating in part the global emittance growth observed before the focussing action.

\section{CONCLUSIONS}

We observe an increase of the electron beam emit. tance $\epsilon_{n}$, immediately after the gun and a subsequent reduction to a rather broad minimum (in some cases there is a gentle asymptotic decrease) located inside the linac sections. This behavior of $t_{n}$ reflects the interplay of focusing, drifting and defocusing space charge fields [7]. The net result is to close the fan in phase space which results in a decrease of the global emittance of the beam. If at this point the beam is accelerated, then the phase spaces of the slice ellipses are locked in with respect to each other due to the decrease of the sface charge forces $\left(F_{\perp} \approx O\left(\frac{1}{\gamma^{2}}\right)\right)$. The electron beam emerging from the linac exhibits a high-brightness characterized by, $B=\frac{l_{p}}{\pi \in N_{x} e_{N . y}}=6 \times 10^{1.1}$

We give in Table I a summary of the parameters for the beam entering the high-energy transport system foliowing the linacs. We find that the optimum photocathode plane to linac entrance distance for our initial conditions is $70 \mathrm{~cm}$.

\section{ACKNOWLEDGEMENT}

The authors wish to thank $D$. Yeremian and $R$. Miller of SLAC for providing the PARMELA input parameters for the traveling-wave accelerating sections. This research was supported by the U.S. Department of Energy under Contract No. DE-ACO2-76-CH00016.

TABLE I. Parameters used in the PARMELA simulations for an axially symmetric $1 \frac{1}{2}$-cell gun in a $\pi$-mode configuration with a solenoid pair.

\begin{tabular}{lc}
\hline \hline RF frequency [MHz] & 2856 \\
Radius of aperture [cm] & 1.0 \\
Field on cathode [MV/m] & 100.0 \\
Lnitial phase [degree] & 43.0 \\
Laser spot sise [mm] $^{*}$ & 0.9 \\
Laser pulse full width" [ps] & 10.0 \\
Charge [nC] & 1.0 \\
B $_{0}[\mathrm{kG}$ ] & 2.2 \\
A verage linac accelerating gradient [MV/m] & 7.0 \\
$\epsilon_{n}, \pi$ mm-mrad & 0.75 \\
dp/p [\%] & 0.2 \\
\hline
\end{tabular}

Uniform profile 
(1) K. Batchelor, et al, The Brookhaven Accelerator Test Facility, Proceedings of the 1988 Linear Acceleration Conierence, CEBAF report 89-001, 540 (1988)

(2) K.-J Kim, Nucl Instr. and Meth. A275, 201 (1989).

(3) Juan C. Gallardo and Robert B. Palmer, Nucl Instr. and Meth A304, 345 (1991)

[4] L. M. Young, private communication.

(5) K Batchelor, et al, Performance of the Brookhaven pho. tocathode rf gun, Proceedings of the Thurteenth International Free Electron Conference, Santa Fe, NM, USA, August 25-30, 1991, Nucl. Instr and Meth A318, 372 (1992).

[6] K R. Crandall, TRACE 3-D Documentation, Los Alamos Accelerator Code Group, LA-UR-90-4146 (1990)

[7] B.E. Carlsten, New photoelectric injector design for the Los Alamos National Laboratory XUV FEL accelerator , Nucl. Instr. and Meth. A285, 313 (1989)

FIG. 1. Normalized trangverse emittance $\epsilon_{n}$ vs. distance $z$ utilizing a solenoid and quadrupole triplet

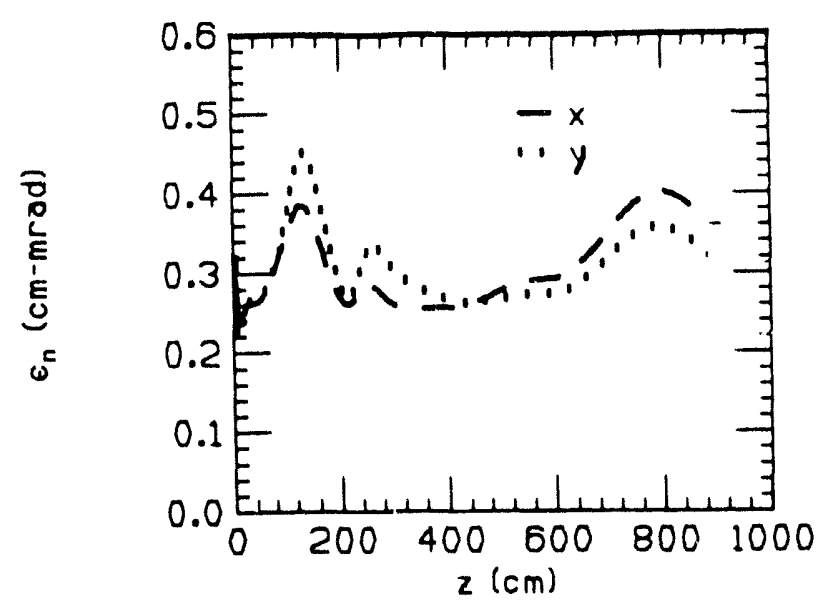

FIG. 2. Normalised transverse emittance en vs. distance a utilising only a solenoid pair-the plane of the photocathode is $62 \mathrm{~cm}$ from the linac entrance

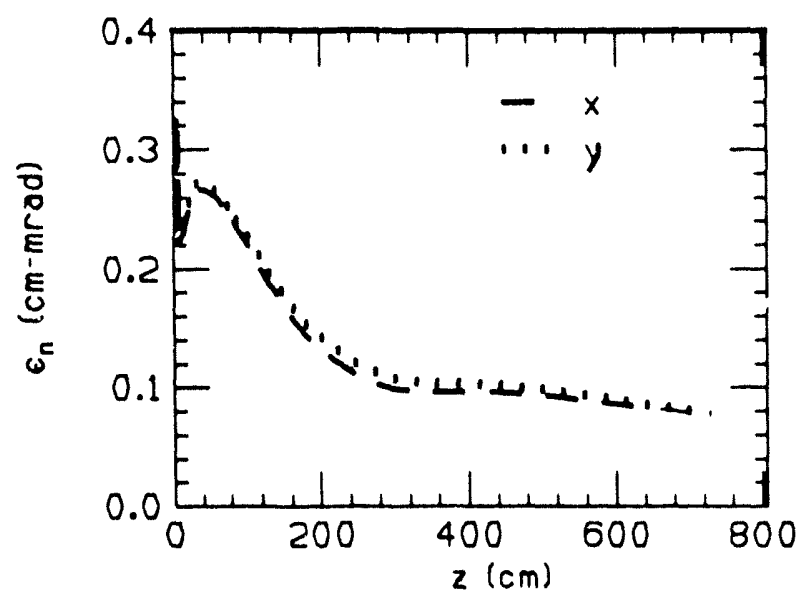

FIC 3. The dp and normalized emittance of the electron beam exiting the $t$ wo linar sections as a function of phase rel. atuve to the electron beam launch phase at the photocathode

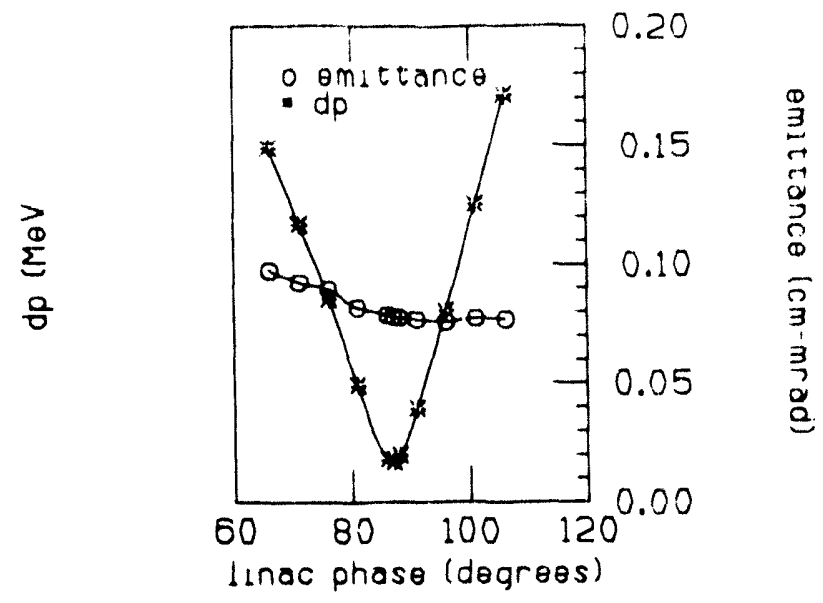

FIG. 4. The normalized emittance of the electron beam exiting the linac sections as a function of the longitudinal distance between the photocathode plane and the linac entrance

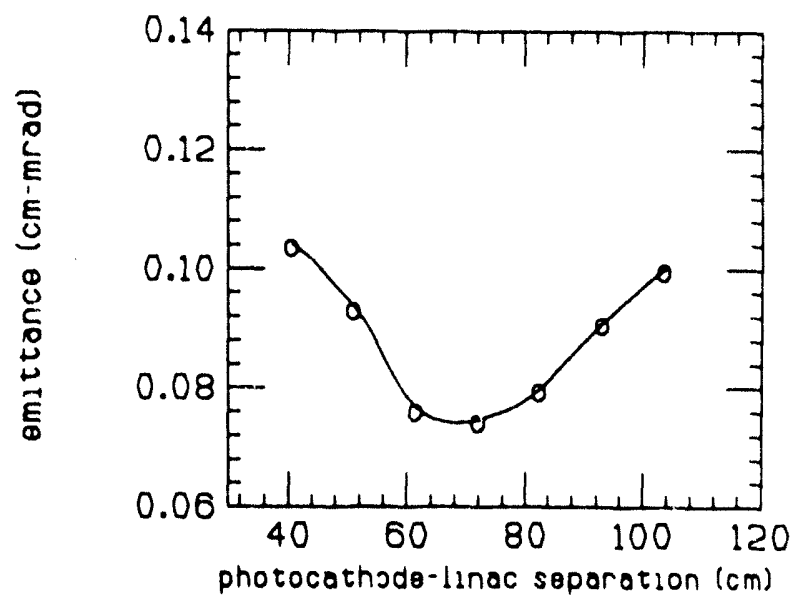

FIG. 5. The trajectories of the end and center slices of the electron beam in $x-x^{\prime}$ phase space

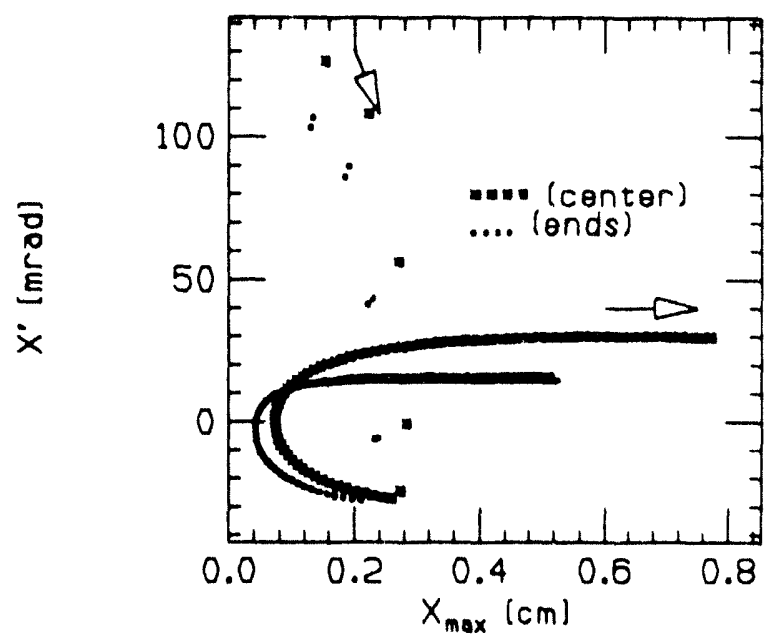



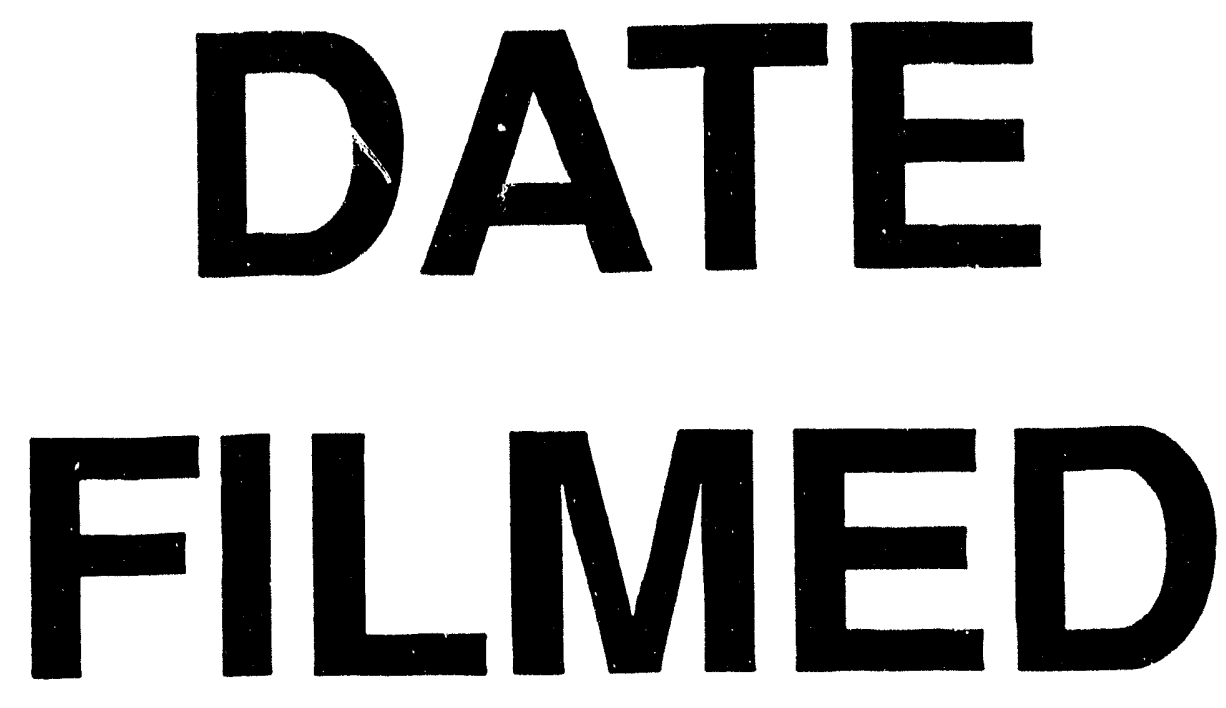

$11 / 10 / 93$
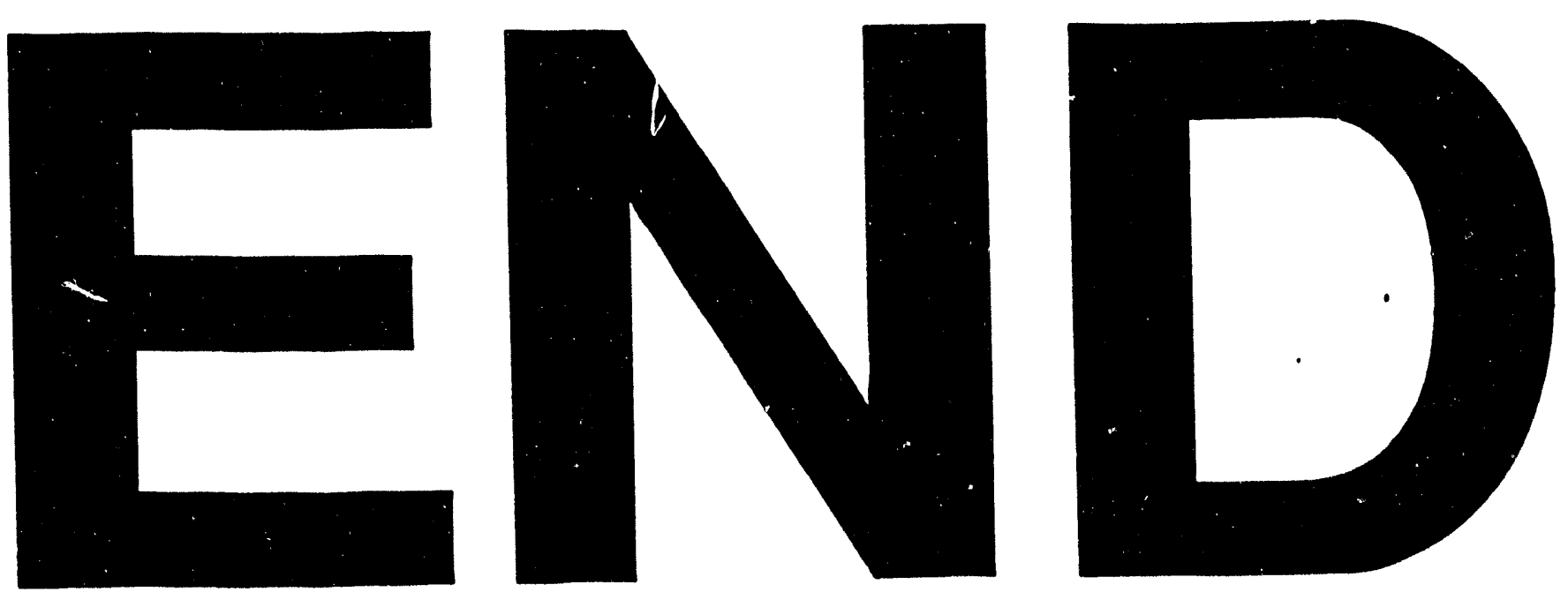
